\title{
FuelCell2009-85182
}

\section{WATER MANAGEMENT IN PEM FUEL CELL - A LATTICE-BOLTZMANN MODELING APPROACH}

\author{
Shiladitya Mukherjee \\ CFD Research Corporation \\ Huntsville, AL, USA \\ Kunal Jain \\ ESI Group \\ Huntsville, AL, USA
}

\author{
J. Vernon Cole \\ CFD Research Corporation \\ Huntsville, AL, USA \\ Ashok Gidwani \\ CFD Research Corporation \\ Huntsville, AL, USA
}

\begin{abstract}
In Proton Exchange Membrane Fuel Cells (PEMFCs), water management and the effective transport of water through the gas-diffusion-layer (GDL) are key issues for improved performance at high power density and for durability during freeze-thaw cycles. The diffusion layer is a thin $(\sim 150-350 \mu \mathrm{m})$, porous material typically composed of a web of carbon fibers and particles, and is usually coated with hydrophobic Teflon to remove the excess water through capillary action. In-situ diagnostics of water movement and gas-reactant transport through this thin opaque substrate is challenging. Numerical analyses are typically based on simplified assumptions, such as Darcy's Law and Leverett functions for the capillary pressure. The objective of this work is to develop a high fidelity CFD modeling and analysis tool to capture the details of multiphase transport through the porous GDL. The tool can be utilized to evaluate GDL material design concepts and optimize systems based on the interactions between cell design, materials, and operating conditions. The flow modeling is based on the Lattice Boltzmann Method (LBM). LBM is a powerful modeling tool to simulate multiphase flows. Its strength is in its kinetic theory based foundation, which provides a fundamental basis for incorporating intermolecular forces that lead to liquid-gas phase separation and capillary effects without resorting to expensive or ad-hoc interface reconstruction schemes. At the heart of the solution algorithm is a discrete form of the well-known Boltzmann Transport Equation (BTE) for molecular distribution, tailored to recover the continuum Navier-Stokes flow. The solution advances by a streaming and collision type algorithm, mimicking actual molecular physics, which makes it suitable for porous media involving complex boundaries. We developed a numerical scheme to reconstruct various porous
\end{abstract}

GDL microstructures including Teflon loading. Single and multiphase LBM models are implemented to compute permeability. Predicted values are in good agreement with measured data. The present modeling approach resolves the GDL microstructures and captures the influence of fiber orientation on permeability and the influence of Teflon loading on the development of preferential flow paths through the GDL. These observations can potentially guide the development of novel GDL materials designed for efficient removal of water.

\section{INTRODUCTION AND SIGNIFICANCE}

Proton Exchange Membrane Fuel Cells (PEMFCs) require effective water management. Water is formed at the cathode catalyst layer that may saturate the adjacent porous gasdiffusion-layer (GDL) thereby, blocking reactant flow from gas-channels. Hence, GDLs are typically coated with hydrophobic medium to removes excess water by capillary action. However, there is limited understanding of water transport inside a GDL. In-situ diagnostics are challenging as the medium is thin $(200-300 \mu \mathrm{m})$ and optically opaque (graphite). Numerical modeling efforts typically use fitting to Darcy's Law for permeability and Leverett function for capillary pressure. Hence, there is significant interest in developing predictive models for transport in GDLs and related porous media. Such models could be applied to analyze and optimize systems based on the interactions between cell design, materials, and operating conditions, and could also be applied to evaluating material design concepts.

In this work, we employ the Lattice-Boltzmann Method (LBM), a modeling approach based on simplified form of 
Boltzmann Transport Equation (BTE), to simulate flow through numerically reconstructed GDLs. LBM has been shown as an effective numerical technique to simulate multiphase and porous media flows [1-6]. We will present an overview of the single and multiphase LBM models as implemented, verification studies for both microstructure reconstruction and transport simulations, and application to single- and two-phase transport in GDL structures. The application studies are designed to both improve understanding of transport within a given structure, and to investigate possible routes for improving material properties through microstructure design.

\section{THEORY AND MATHMATICAL FORMULATION}

In this section, the multiphase LBM flow model with surface wettability effect is discussed. This is followed by description on the GDL reconstruction scheme.

\section{The Lattice-Boltzmann Model (LBM)}

LBM is based on solution of Boltzmann transport equation given by,

$$
\frac{D f(\mathbf{x}, \xi, t)}{D t}=\frac{\partial f}{\partial t}+\xi \cdot \nabla f+\mathbf{F} \cdot \nabla_{\xi} f=\left(\frac{\partial f}{\partial t}\right)_{\text {coll }}
$$

Here, $f$ is the molecular density distribution function defined as

$$
\begin{aligned}
& \rho(\mathbf{x}, t)=\int f(\mathbf{x}, \xi, t) d \xi \\
& \rho(\mathbf{x}, t) \mathbf{u}(\mathbf{x}, t)=\int f(\mathbf{x}, \xi, t) \xi d \xi
\end{aligned}
$$

where $\rho$ is the density, $\rho \mathbf{u}$ momentum at location $\mathbf{x}$ at time $t$, and $\xi$ the molecular velocity. $\mathbf{F}$ is the mean force field. The right side of the equation is the rate of change in $f$ due to intermolecular collisions. In LBM, the collision operator is modeled by Bhatanagher-Gross-Krook (BGK) model,

$$
\left(\frac{\partial f}{\partial t}\right)_{\text {coll }}=-\frac{f-f^{e q}}{\lambda}
$$

The model assumes that particle distributions $f$ approach local equilibrium $f^{e q}$ linearly over a characteristic time $\lambda$. This characteristic time is related to fluid kinematic viscosity $v$. While simple, this model has well-known limitations. In particular, for porous media flows employing the BGK model results in viscosity dependence of computed permeability. We have also reconfirmed this observation through our own set of simulations. Hence, in this work a multiple-relaxation-time (MRT) collision model is implemented. In the MRT model, the single relaxation time $\lambda$ is replaced by a collision-matrix given by,

$$
\left(\frac{\partial f_{\alpha}}{\partial t}\right)_{\text {coll }}=-\Lambda_{\alpha \beta}\left(f_{\beta}-f_{\beta}^{e q}\right)
$$

Here, $\alpha, \beta$ corresponds to particle velocities $\xi_{\alpha}$ and $\xi_{\beta}$, respectively. Employing the collision-matrix allows separation of relaxation time-scales between hydrodynamic modes such as velocity, pressure and stress-tensors, thus improving numerical accuracy and stability.

\section{Multiphase LBM}

We implemented the multiphase LBM model of He et al. [7]. In this model, $\mathbf{F}$ is an interfacial force formulated as function of higher order density gradient. It is sum of a phase segregation and surface tension force. The phase segregation force is related to gradient of the non-ideal part of the equationof-states (EOS) which separates fluid into respective liquid and gas phase densities by entropy minimization. The surface tension force depends on the interfacial curvature, and is scaled by the strength of the molecular interaction.

To improve numerical stability two separate distribution functions are used. One distribution function $f$ is for a phasetracking variable, known as the index-function $\varphi$. The transport equation of $f$ recovers an advection-diffusion equation known as the Cahn-Hilliard equation. Transition of $\varphi$ from the limiting values at the liquid to gas phase occurs over finite number of lattice nodes. The interface is implicitly defined as the contour of $\varphi$ having the average of these limiting values. Fluid properties, such as, density and viscosity are interpolated from $\varphi$. A second distribution function $g$ is used to compute pressure and momentum. The transport equation of $g$ recovers the Navier-stokes equation with a surface tension term. The surface tension is computed from $\varphi$ as proportional to $\varphi \nabla\left(\nabla^{2} \varphi\right)$. In this work, the first and second-order derivatives are computed as weighted sum of the second-order central difference along the lattice-velocity directions. The limiting values of $\varphi$ are obtained analytically from the EOS and can be further refined from the simulation of liquid film or drop equilibration.

\section{Surface Wettability Model}

The wettability model of Yiotis et al. [8] is employed. The model assigns a $\varphi$ value to the solid node to modify capillary forces near the solid boundary. The resulting contact angle scales linearly from $0^{\circ}$ to $180^{\circ}$ as $\varphi$ is varied from its limiting value in liquid phase to gas phase.

\section{Microstructure Reconstruction Model}

The GDL microstructure of non-woven carbon paper is generated by laying fixed diameter, continuous, cylindrical fibers in parallel planes. Within a plane the fibers are randomly orientated and may intersect each other. This approach is similar to that reported by Schulz et al. [9]. In this work, spacing between parallel planes are equal to fiber diameter $f_{d}$. The maximum inclination angle from the plane is assumed $10^{\circ}$. 


\section{MODEL VALIDATION}

The LBM implementation and GDL structure generation is benchmarked against numerous test cases reported in the following references. In Figure 3, a test case is shown for flow through an idealized porous structure of simple-cubic (SC) arrangement of solid spheres. Permeability $\kappa$ is computed from the average flow velocity $u$ by applying Darcy's law,

$$
u=\frac{\kappa}{\mu \varepsilon}\left(\frac{P_{\text {in }}-P_{\text {out }}}{L}\right)
$$

Here, $\mu$ is the viscosity, $\varepsilon$ the porosity, $P_{\text {in }}$ the inlet pressure, $P_{\text {out }}$ the outlet pressure, and $L$ the channel length. In Table 1, computed permeability is compared with the analytical solution of Chapman and Higdon [10] at different porosity by varying the sphere size. The values are in agreement within 5\% of the analytical solution.

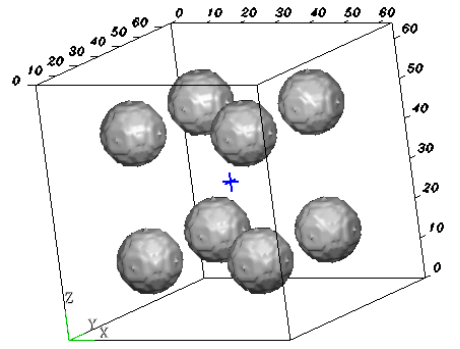

(a) Solid spheres

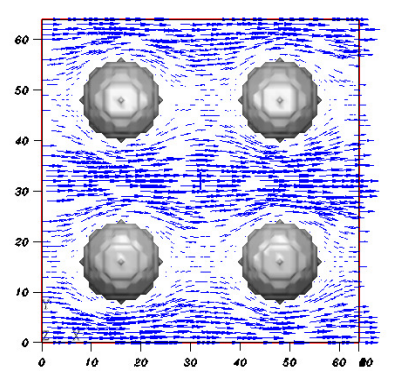

(b) Flow around spheres
Figure 1. Flow through simple-cubic arrangement of solid spheres.

\begin{tabular}{|c|c|c|c|}
\hline $\begin{array}{c}\text { Porosity, } \\
\varepsilon\end{array}$ & $\begin{array}{c}\text { Analytical, } \\
\mathrm{\kappa} / \mathrm{d}^{2}\end{array}$ & $\begin{array}{c}\text { Computed, } \\
\mathrm{\kappa} / \mathrm{d}^{2}\end{array}$ & \% Error \\
\hline 0.992 & 0.2805 & 0.2843 & 1.3 \\
\hline 0.935 & 0.0761 & 0.0749 & 1.6 \\
\hline 0.779 & 0.0192 & 0.0189 & 1.6 \\
\hline 0.477 & 0.0025 & 0.0026 & 4 \\
\hline
\end{tabular}

Table 1. Non-Dimensional Permeability of Simple-Cubic (SC) Arrangement of Spheres.

GDL microstructure reconstruction is validated by comparing computed pore size distribution and permeability with measurements. In Figure 2, reconstructed Toray090 is shown with pore size distribution. The peak in the pore volume is at radius $11 \mu \mathrm{m}$. This is consistent with reported measured value of $12 \mu \mathrm{m}$. In Table 2 , in-plane and through-plane permeability are computed for Toray090 and SGL10BA. GDLs are $200 \mu \mathrm{m}$ thick with spatial resolution of $3.4 \mu \mathrm{m}$, porosities 0.78 and 0.88 , respectively. SGL10BA has higher permeability than Toray090 due to higher porosity. The in-plane permeability is greater than through-plane value. The difference is due to the preferred fiber orientation in the material plane and suggests that alignment to the mean-flow direction have less resistance to the flow. The computed trends are consistent with these observations and are quantitatively similar to those reported in the numerical work of Schulz et al. [9].

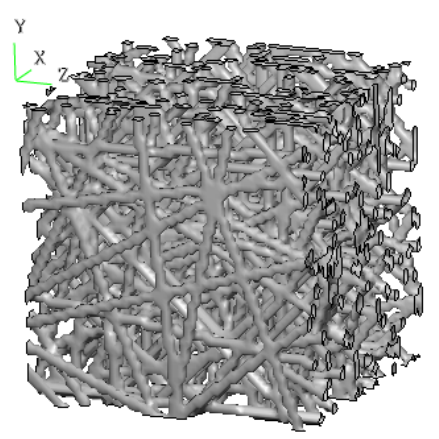

(a) Carbon paper

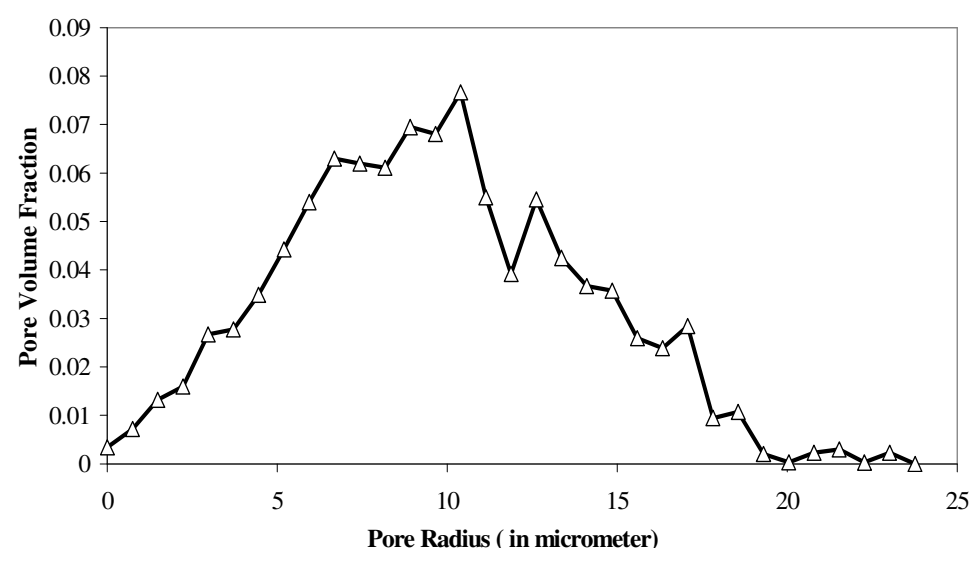

(b) Pore size distribution

\begin{tabular}{|l|c|c|c|}
\hline Material & $\begin{array}{c}\text { Measured } \\
\left(10^{-12} \mathrm{~m}^{2}\right)\end{array}$ & $\begin{array}{c}\text { Simulation } \\
\left(10^{-12} \mathrm{~m}^{2}\right)\end{array}$ & $\%$ Error \\
\hline $\begin{array}{l}\text { Toray090 } \\
\text { (Through-plane) }\end{array}$ & 8.3 & 6.241 & -24.8 \\
\hline Toray090 (In-plane) & - & 8.647 & - \\
\hline $\begin{array}{l}\text { SGL10BA } \\
\text { (Through-plane) }\end{array}$ & 18 & 21.71 & 20.6 \\
\hline SGL10BA (In-plane) & 33 & 30 & 9.1 \\
\hline
\end{tabular}

Table 2. Carbon-Paper Microstructure Permeability.

\section{RESULTS AND DISCUSSION}

In this section, we first report influence of GDL microstructure on gas-permeability. Please refer to the earlier works by Mukherjee et al. [5,6] for comprehensive discussion. Next, we present simulation results of multiphase water transport through the GDL, which is the main focus of this paper. Specifically, we investigate distribution of water at 
various liquid saturations, and its transport behavior when Teflon is added.

\section{Influence of GDL Microstructure on Gas-Permeability}

\section{Preferential Fiber Orientation}

In Figure 3, influence of GDL fiber orientation on permeability is shown. Three cases compared are when fibers are constrained to orient within $5^{\circ}, 45^{\circ}$ and $90^{\circ}$ of an in-plane direction (y-axis).
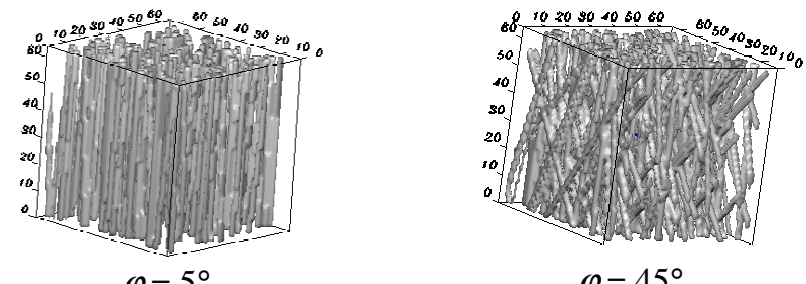

$\varphi=5^{\circ}$

$\varphi=45^{\circ}$
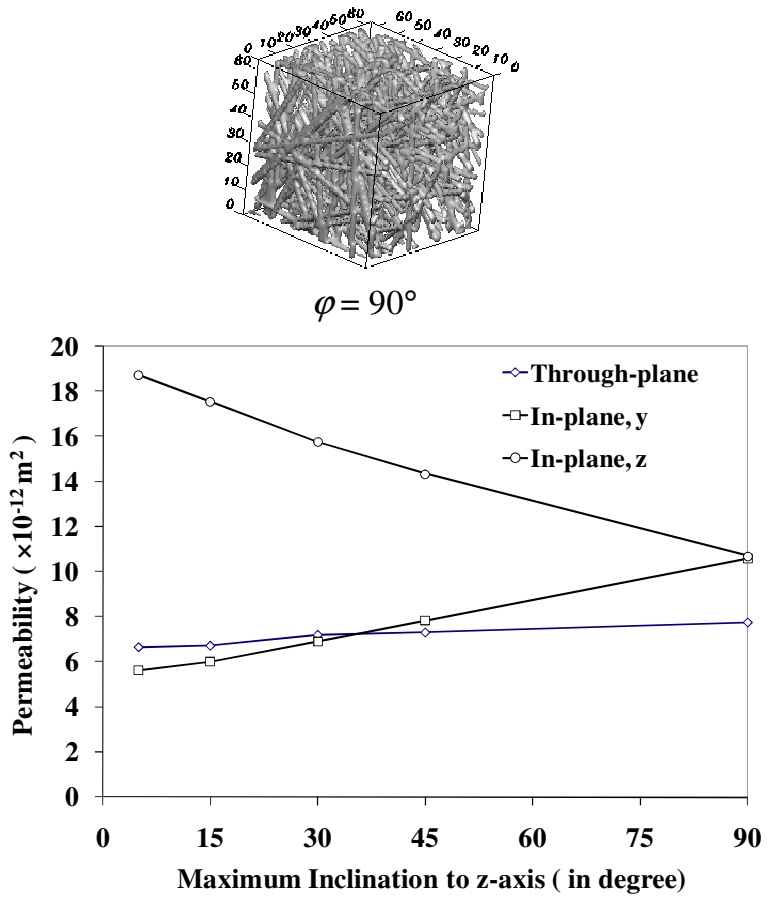

Figure 3. Through and In - plane permeability at various fiberorientations.

The through-plane permeability is less sensitive and decrease by about $15 \%$, while in-plane permeability increases by about $75 \%$ in the alignment direction and decreases by $47 \%$ in the normal direction. The results suggest that the in-plane permeability can be increased significantly along a preferred direction by orienting the fibers along it, without resulting in significant drop in through-plane permeability. This information can be used to enhance cross-channel gas-diffusion in GDL. For example, GDL-material can be constructed in two layers. The gas-channel side consisting of fibers with preferred orientations, assisting in-plane gas flow between neighboring gas-channels, and the catalyst side having fibers randomly oriented thereby allowing uniform distribution of incoming gas on the reaction sites.

\section{External Compression}

GDL under external compression is modeled following the same approach as by Schulz et al. [9] i.e. by linearly scaling the height of the solid voxels from the base of the material. Figure 4 shows Toray090 at compression ratio, $\mathrm{c}=0.8$ and 0.6 .
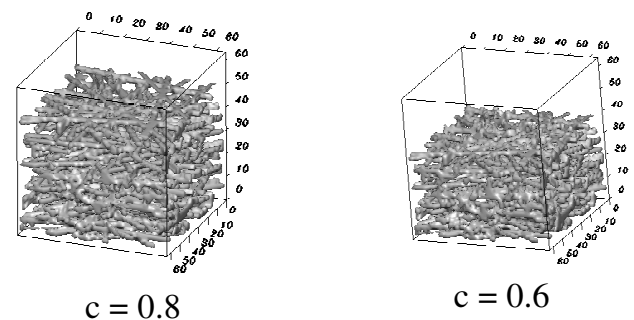

$\mathrm{c}=0.6$
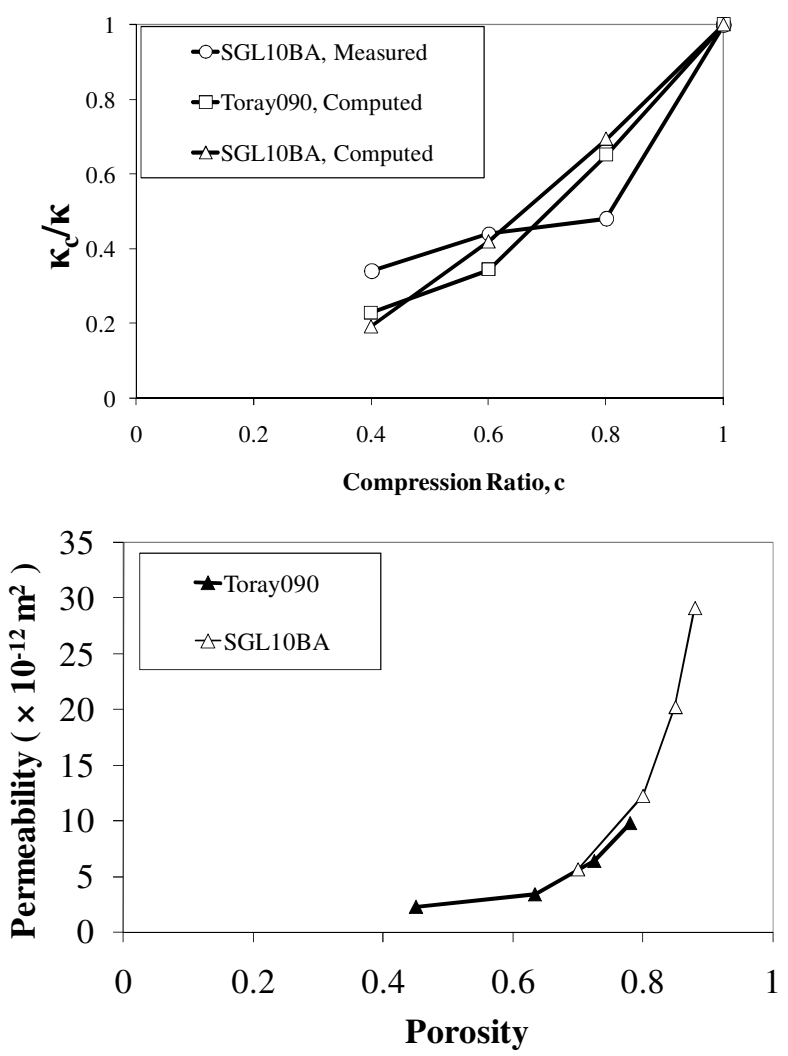

Figure 4. Permeability of Toray090 and SGL10BA under compression.

Computed through-plane gas-permeabilities are shown at different compression ratio for Toray090 and SGL10BA. The measured data is by Dohle et al. [10] on SGL10BA. As compression ratio decreases, GDL porosity decrease, hence, 
permeability decreases. Computed and measured data are consistent with this expectation.

\section{Multiphase Transport in GDL}

Liquid Drainage/Imbibitions in a Capillary Tube

Figure 5 shows liquid drainage and imbibitions due to capillary action. The capillary tube initially half filled and connected to a liquid reservoir maintained at the same pressure as the tube exit. The liquid drains out of the tube when contact angle $\theta>90^{\circ}$ (hydrophobic), fills in the tube when $\theta<90^{\circ}$ (hydrophilic), and remains stationary when $\theta=90^{\circ}$.

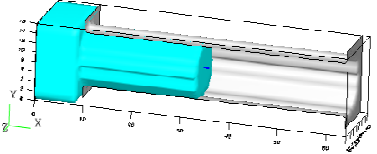

(a) Initial set-up

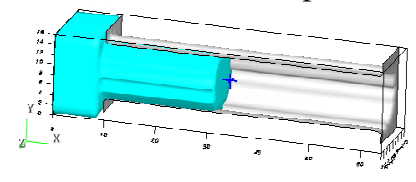

(c) $\theta=90^{\circ}$

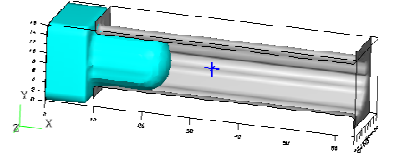

(b) $\theta=125^{\circ}$

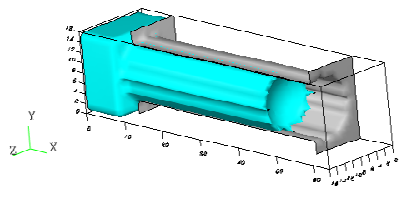

(d) $\theta=75^{\circ}$
Figure 5. Liquid drainage and imbibition due to capillary action in a tube.

Influence of Liquid Saturation on Water Distribution

Figure 6 shows water distribution in Toray-050. The GDL is $170 \mu \mathrm{m}$ thick, cross-sectional area is $200 \mu \mathrm{m} \times 200 \mu \mathrm{m}$, porosity 0.75 , contact angle $144^{\circ}$. The grid resolution is $3.4 \mu \mathrm{m}$. The inlet boundary is no-slip wall. The space between GDL and inlet is $70 \mu \mathrm{m}$ thick, initially filled with liquid corresponding to liquid saturation of $\alpha=1$. The outlet is an open boundary at standard atmospheric pressure. The space between GDL and inlet is $70 \mu \mathrm{m}$ thick, initially filled with gas corresponding to liquid saturation of $\alpha=0$.

Initially, the GDL is filled with a uniform saturation $0<\alpha$ $<1$. As the time progresses phase separation sets in. In the figure liquid distribution is shown without showing the fibers for clarity. At low saturation $(\alpha=0.3)$ globules of droplets are formed that are distributed throughout the GDL. Most of the droplets are separated from each other. At higher liquid saturation $(\alpha=0.4)$, the droplets coalesce and form interconnected liquid streams. Part of the liquid also remains as isolated droplets. At still higher liquid saturation $(\alpha=0.7)$ liquid emerges through multiple openings at the GDL surface. Interconnected liquid paths are visible from inlet to these openings.

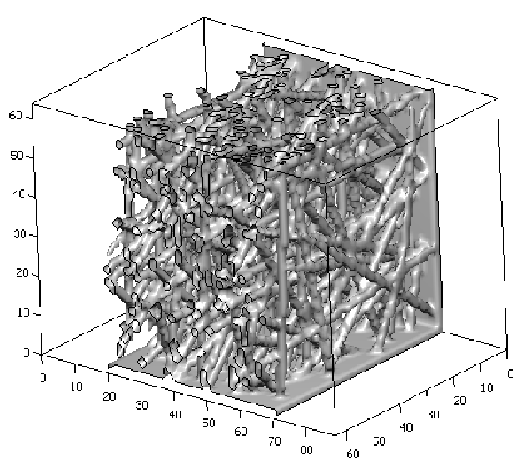

(a) Toray050

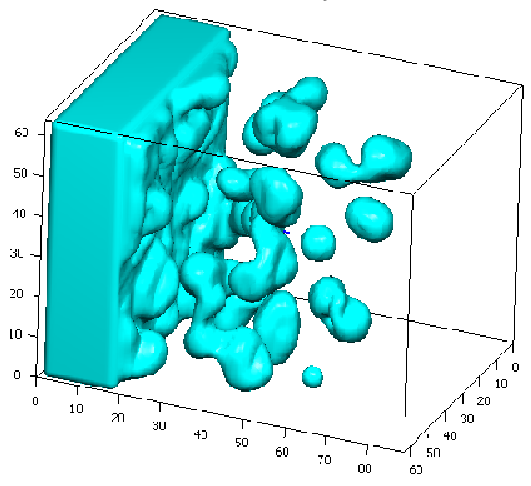

(b) $\alpha=0.3$

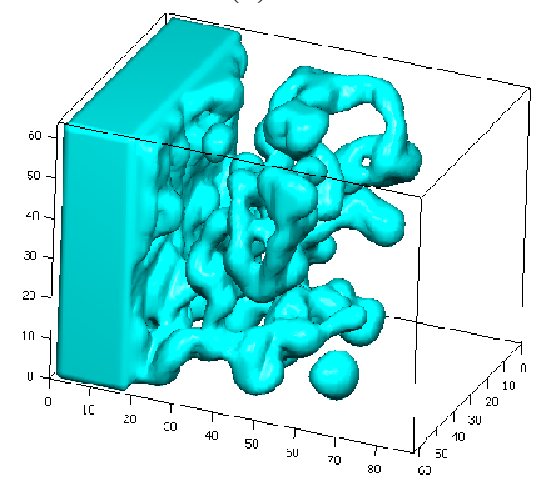

(c) $\alpha=0.4$

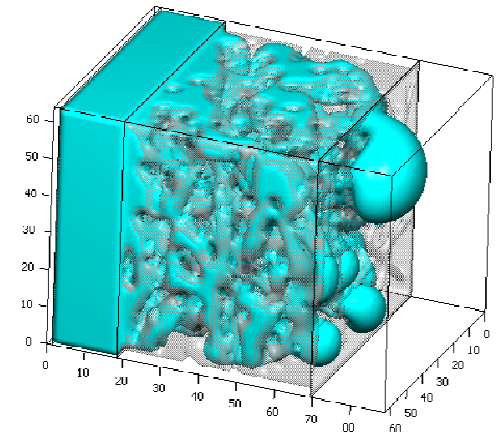

(d) $\alpha=0.7$

Figure 6. Water Distribution inside wet GDL. The material is Toray- 050 . 


\section{Capillary Pressure-Saturation Curve}

To obtain a capillary pressure saturation curve, a hydrophobic medium of porosity 0.5 is positioned at the GDL outlet. The pores are $3.4 \mu \mathrm{m}$ in diameter and uniformly distributed. The layer prevents liquid to escape through it, but the gas can flow. Liquid distribution in the presence of the hydrophobic layer is shown in Figure 7(a) at $\alpha=0.7$. Compared to Figure 6(d), there is no emerging droplet from the GDL surface. The pressure-saturation $\left(\mathrm{P}_{\mathrm{c}}-\alpha\right)$ plot is shown in Figure 7 (b). The leveret function is plotted using following values for surface tension water $\sigma=0.072 \mathrm{~N} / \mathrm{m}$ and Toray-050 permeability 4 Darcy i.e. $4 \times 10^{-12} \mathrm{~m}^{2}$ [Measured by Ballard Power Systems].

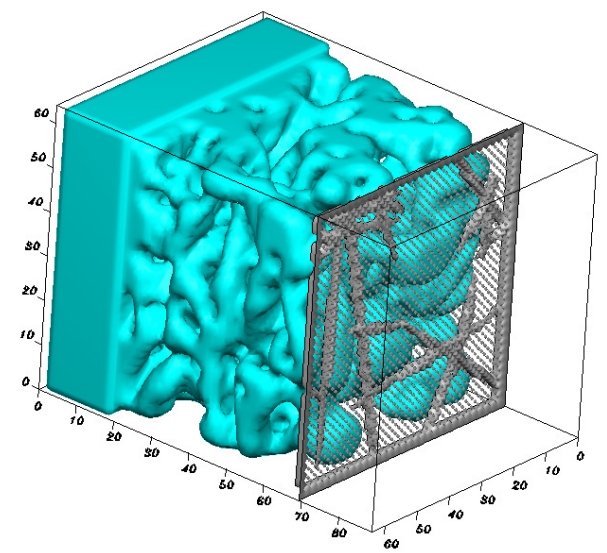

(a) $\alpha=0.7$, Hydrophobic plug at GDL exit

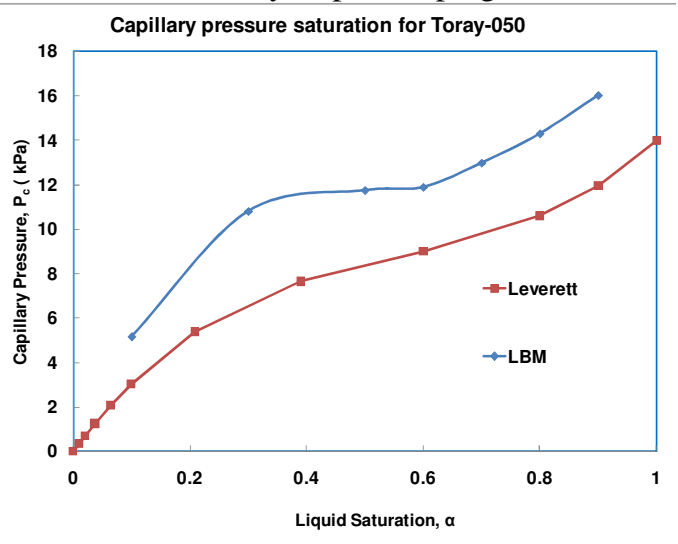

(b) Capillary pressure-saturation curve

Figure 7. Comparison of computed capillary pressure-saturation with Leverett function.

Computations are in good agreement with measurements qualitatively. However, the computed capillary pressure over predicts leveret function. The difference may be due to assumption of fixed contact angle in the LBM simulation. This contact angle is based on experimentally reported value measured on GDL external surface. However, internal GDL contact angle values may be different due to pore morphology, partial coating of fibers by Teflon. Further investigation is needed for appropriate selection of internal contact angle in the simulation.

\section{Fiber Wettability Effect on Water Transport}

Next, we demonstrate influence of fiber wettability on liquid flow pattern. The material is Toray090, $200 \mu \mathrm{m}$ thick comprising of 25 layers of carbon fibers of diameter $8 \mu \mathrm{m}$, the in-plane area is $218 \mu \mathrm{m} \times 218 \mu \mathrm{m}$ and porosity 0.78 . An open space of thickness $109 \mu \mathrm{m}$ is provided at the gas-channel side to allow liquid-breakthrough and formation of multiple droplets. Liquid is introduced at the boundary on the catalyst side. The side-boundaries are no-slip walls of neutral wettability i.e. $\theta=$ $90^{\circ}$. In Figure 8, snap-shots of liquid-breakthrough are shown at different fiber hydrophobicity. Liquid breaks through the channel side of the GDL in the form of droplets. These droplets may coalesce forming larger drops. As hydrophobicity is increased liquid saturation at break-through decreases. Liquid flows as small streams leading to surface pores. These observations are consistent with other published data [12].

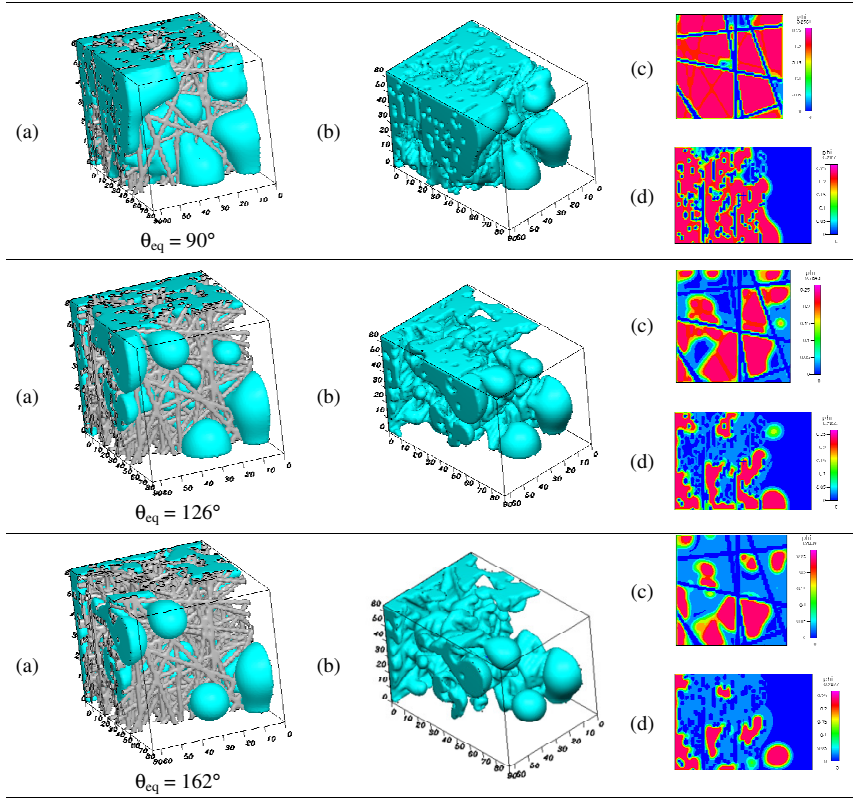

Figure 8. Influence of fiber wettability on liquid saturation as it breaks through the porous gas-diffusion layer (GDL). (a)

Emergence of liquid drops at the GDL and gas-channel interface. (b) Liquid distribution inside the porous medium. (c) Liquid-gas distribution on a plane normal to the flow. (d) Liquid-gas distribution on a plane parallel to the flow. Liquid saturation decreases as fiber hydrophobicity increases.

\section{Teflon Loading Effect on Water Transport}

Teflon Loading Model: Hydrophobic Teflon loading is modeled by identifying spatial distributions of pores of different sizes and successively filling fluid voxels corresponding to the smallest pore sizes to match Teflon $\%$. Thus, Teflon is distributed to smaller pores and corners of the larger pores. Increase in fiber diameter due to Teflon coating is 
assumed negligible. However, their wettability is changed from hydrophilic to hydrophobic. In Figure 1, numerically generated microstructure of TGP-H-050 is shown in the range 0-60\% Teflon loading. The GDL thickness is $170 \mu \mathrm{m}$, area $102 \mu \mathrm{m} \times$ $102 \mu \mathrm{m}$, fiber diameter $f_{d}=8 \mu \mathrm{m}$. Porosity of the untreated GDL is 0.75 .

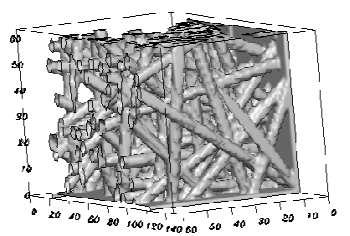

(a) $0 \% \mathrm{PTFE}$

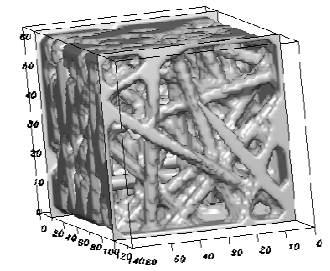

(c) $20 \%$ PTFE

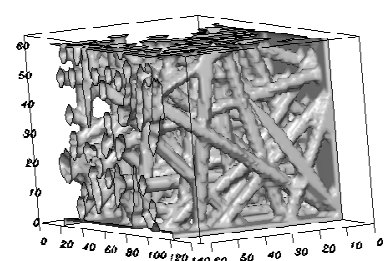

(b) $5 \%$ PTFE

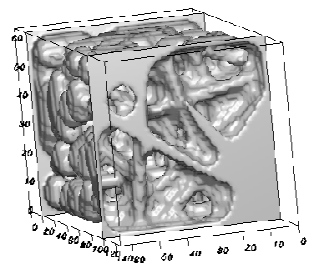

(d) $60 \%$ PTFE
Figure 9. Numerically generated Toray 050 microstructure at various Teflon (PTFE) loading.

Model Validation: As Teflon \% increases, gaspermeability $\kappa$ is expected to decrease due to lower porosity. In Figure $3, \kappa$ is plotted at different Teflon loading. The computed permeability values are qualitatively consistent with measurements by Ballard Power Systems. Quantitatively they are in agreement within $15 \%$ up to Teflon loading of $30 \%$. However, at higher Teflon loading the difference increases.

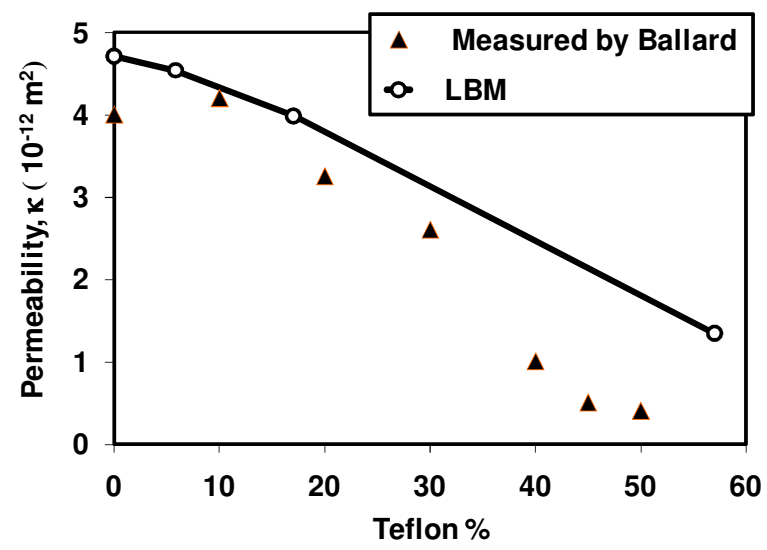

Figure 10. Toray050 through-plane permeability at various Teflon loading.
This suggests that up to Teflon loading of $30 \%$, the above model for Teflon distribution is adequate. At still higher Teflon $\%$ additional modeling refinements are needed.

Multiphase Transport: Liquid flow through interconnected streams becomes more evident as Teflon loading is increased. In Figure 6, liquid distribution is shown at 0, 20 and $40 \%$ Teflon.
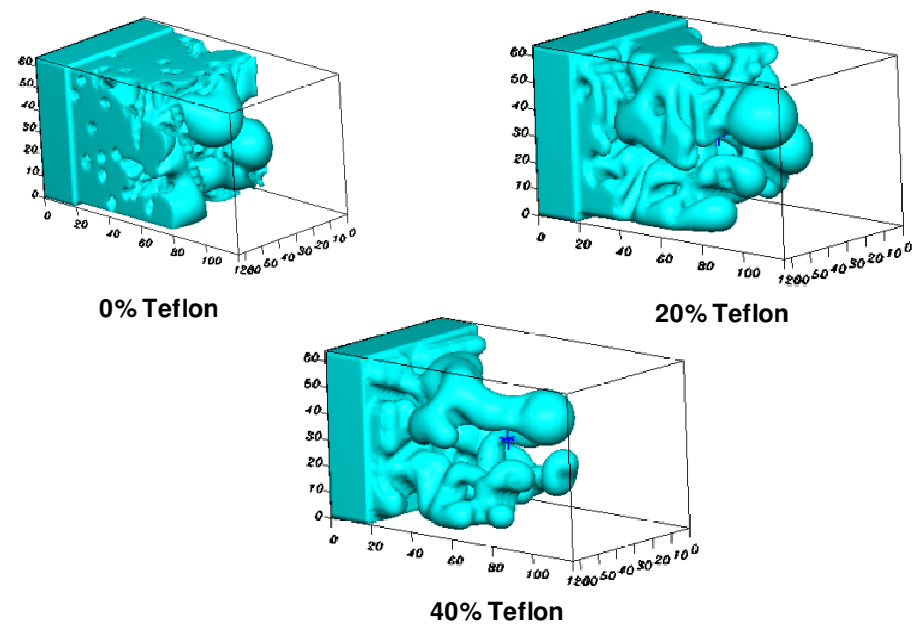

Figure 11. Liquid flowing in interconnected streams as Teflon $\%$ is increased.

As Teflon is added there is stronger repulsion to liquid in small pores due to capillary forces. Therefore, liquid flows primarily through interconnected larger pores. Hence, preferential liquid flow paths and preferred spots on GDL surface for droplet inception are observed when Teflon $\%$ is increased.

\section{SUMMARY AND CONCLUSIONS}

LBM is shown is an effective numerical tool in simulating flow through gas-diffusion-layer of PEM Fuel Cells. The GDL microstructure can be modeled to incorporate effects of fiber orientation, external compression, fiber wettability and Teflon loading. Computed permeabilities are qualitatively in agreement with measured or expected trends. Simulation results also provide insight into GDL in-situ water distribution. At lower saturation liquid is in the form of isolated droplets. At higher saturation these globules get connected and immerge as droplets from preferred sites on the GDL surface. As Teflon content is increased liquid break-through is observed at lower saturation. The results are encouraging as it demonstrates that LBM can be used to improve understanding of transport within a given GDL microstructure, and investigate possible routes for improving material properties through microstructure design. 


\section{ACKNOWLEDGMENTS}

This material is based upon work supported by the Department of Energy under award number DE-FG3607G017010.

Disclaimer: This report was prepared as account of work sponsored by an agency of the United States Government. Neither the United States Government nor any agency thereof, nor any of their employees, makes any warranty, express or implied, or assumes any legal liability or responsibility for the accuracy, completeness, or usefulness of any information, apparatus, product, or process disclosed, or represents that its use would not infringe privately owned rights. Reference herein to any specific commercial product, process, or service by trade name, trademark, manufacturer, or otherwise does not necessarily constitute or imply its endorsement, recommendation, or favoring by the United States Government or any agency thereof. The views and opinions of authors expressed herein do not necessarily state or reflect those of the United States Government or any agency thereof.

\section{REFERENCES}

1. Mukherjee S. and Abraham, J. (2007), J. Coll. Inter. Sci., 312, 341.

2. Mukherjee S. and Abraham, J. (2007), Phys. Fluids, 19, 052103.

3. Mukherjee S. and Abraham, J. (2007), Phys. Rev. E, 75, 026701.

4. Mukherjee S. and Abraham, J. (2007), J. Computers Fluids, 36, 1149.

5. Mukherjee S., Cole J.V., Jain K., and Gidwani A., "Lattice-Boltzmann Simulations of Multiphase Flows in PEM Fuel Cell GDLs and Microchannels", ECS Transactions, 16(2) 67-77 (2008)

6. Mukherjee S., Cole J.V., Jain K., and Gidwani A., "Lattice-Boltzmann Simulations of Multiphase Flows in Gas-Diffusion-Layer (GDL) of a PEM Fuel Cell", $100^{\text {th }}$ AIChE Annual Meeting, Philadelphia, PA Nov 16-21, 2008.

7. He X., Chen, S. and Zhang, R. (1999), J. Comp. Phys., 152, 642.

8. Yiotis, A.G., Psihogios, J., Kainourgiakis, M. E., Papaioannou, A., and Stubos, A.K. (2007), Coll. Surf. A: Physiochem. Eng. Aspects, 300, 35.

9. Schulz, V. P., Becker, J., Wiegmann, A., Mukherjee, P.P., and Wang, C.Y. (2007), J. Electrochem. Soc., 154, B419.

10. Dohle, H., Jung, R., Kimiaie, N., Mergel, J., and Müller, M. (2003), J. Power Sources, 124, 371.

11. Martys, N.S. and Hagedorn, J.G. (2002), Materials and Structures, 35, 650.
12. Bazylak, A., Sinton, D., and Djilali, N. (2008), Journal of Power Sources, 176, 240-246. 